\title{
ESTIMATIVA DE CHUVAS INTENSAS PARA O OESTE DE MINAS GERAIS E O ENTORNO DO RESERVATÓRIO DA USINA HIDRELÉTRICA DE FURNAS
}

\author{
Marco Aurélio de Mello Machado \\ Professor Depto. de Solos e Engenharia Agrícola \\ Universidade Federal do Paraná (UFPR). Curitiba-PR \\ e-mail: marcomello@ufpr.br
}

Eder David Borges da Silva

Eng. Agrônomo. Pesquisador Júnior I - Instituto de Tecnologia para o Desenvolvimento-LACTEC - Curitiba-PR

e-mail: ederdbs@gmail.com

\section{RESUMO}

Uma precipitação intensa é definida como a chuva cuja intensidade ultrapassa determinado valor em uma dada distribuição espacial e temporal. As variações das chuvas intensas ao longo do espaço geográfico e ao longo do tempo representam um dos principais estudos hidrológicos com aplicações na conservação dos solos e na engenharia de construção e de drenagem. Para uma correta utilização desses dados, faz-se necessário conhecer as relações existentes entre as quatro características fundamentais de uma chuva: intensidade, duração, frequiência e distribuição especialmente agora, quando se projetam cenários de mudanças climáticas, relativas à freqüência e intensidade de eventos extremos. Neste trabalho foram estimadas as equações de chuvas intensas ou as relações intensidade-duração-frequência $(i-d-f)$ para localidades do oeste do estado de Minas Gerais e do entorno do lago do reservatório da Usina Hidrelétrica de Furnas. Foram selecionadas 44 localidades, compreendidas entre $20^{\circ} 00$ e $21^{\circ} 25^{\prime}$ de latitudes Sul e entre $45^{\circ} 00^{\prime}$ a $46^{\circ} 25^{\prime}$ de longitudes Oeste. Foi empregada a metodologia do inverso das potências da distância entre as localidades o que permitiu estimar, para cada uma, os parâmetros da equação $i-d-f$, bem como as chuvas intensas máximas médias (de 5 a 60 minutos), em função de períodos de retorno de 2 a 10.000 anos. Tais estimativas precisam, posteriormente, ser checadas com dados pluviográficos observados, para a verificação do grau de acurácia do método aqui empregado. Contudo, os resultados demonstraram grande potencial de aplicação em áreas da engenharia, bem como em práticas de conservação dos solos e águas.

Palavras-Chave: Hidrologia. Chuvas intensas. Intensidade-duração-frequência.

\section{INTENSE RAINFALL ESTIMATE FOR WEST MINAS GERAIS STATE AND THE VICINITY OF FURNAS HYDROELECTRICAL RESERVOIR}

\begin{abstract}
An intense rainfall is defined as the precipitation with intensity exceeding a value in a spatial and temporal distribution. How variable is its intensity is the subject of most main hydrology studies to be applied in soil conservation and construction and drainage engineering. As to better use the data available, it is necessary to know the relation between four characteristics of the rainfall: intensity,
\end{abstract}


duration, frequency and spatial distribution, mainly in climate change scenarios closely related to extreme weather occurrences. For the purpose of this work, we have estimated intense rainfall equations or intensity-duration-frequency $(i-d-f)$ for sites in western Minas Gerais state and the vicinity of Furnas Plant. We have selected 44 sites between $20^{\circ} 00^{\prime}$ and $21^{\circ} 25^{\prime}$ South and between $45^{\circ} 00^{\prime}$ to $46^{\circ} 25^{\prime}$ West. For all sites we have estimated the $i$ - $d$ - $f$ equation, as well as the average maximum duration of an intense rainfall event (from 5 to 60 minutes), in relation to periods of return between 2 to 10,000 years. This estimate must be cross checked with rainfall graphics so as to conciliate data. However, its potential applicability is to emphasize, especially in some engineering area where soil and water conservation is in place.

Key-words: Hidrology. Intense rainfal. Intense-duration-frequency.

\section{INTRODUÇÃO}

Chuvas intensas são aquelas cuja intensidade ultrapassa determinado valor tomado como referência, podendo variar de alguns poucos minutos até dezenas de horas (PRUSKI et al., 2006). Uma chuva intensa atua de maneira direta sobre os processos erosivos do solo, tanto em áreas urbanas quanto rurais, além de ser um importante elemento de projeto de dimensionamento de sistemas de drenagem e em obras de construção civil, como vertedores de barragens, sistemas de drenagem e galerias de águas pluviais. Além disso, grande parte da agricultura e a maior parcela da matriz energética do país guardam forte dependência do suprimento hídrico no período de verão. Tanto a agricultura de sequeiro quanto usinas hidrelétricas, dependem, para uma produção satisfatória de grãos e de energia, de um período regular e suficiente de chuvas. O não atendimento dessas exigências pode vir a afetar fortemente a produção de alimentos e de energia, impactando a economia local, regional e nacional.

Um evento isolado de precipitação pode ser definido pelas suas quatro características fundamentais: intensidade, duração, frequência e distribuição. Essas relações podem ser obtidas de séries climáticas históricas, a partir da análise de pluviogramas e da distribuição estatística das chuvas quanto aos seus valores máximos anuais, com diferentes durações e sob diversos tempos de retorno. Neste caso, são utilizadas equações empíricas (PRUSKI et al, 2006) que podem trazer erros de ajuste que devem ser avaliados pela comparação com dados observados.

Frequentemente, as séries históricas de precipitação são mais longas do que as séries de vazão, razão pela qual o estudo das precipitações máximas pode ser importante para que se conheça determinada vazão de enchente de uma bacia hidrográfica. (TUCCI, 2004). Contudo, é comum que o regime pluviométrico de determinada região, em especial a intensidade de precipitação, não seja 
conhecido em função da inexistência de informações pluviográficas, o que pode levar à diminuição na confiabilidade dos projetos de engenharia de água e solo.

Nos últimos anos, pesquisas nas áreas de meteorologia e climatologia têm alertado para o aumento no número e na frequência de eventos climáticos extremos. Estes eventos podem estar estreitamente ligados ao aumento na concentração de vapor de água na atmosfera, em função de um provável aumento nas temperaturas superficiais. Como o vapor de água é gás aprisionador de radiação, especialmente de ondas longas, uma maior concentração desse gás representa maior quantidade de energia disponível na atmosfera. Outra das possíveis consequências desse quadro é o aumento da aleatoriedade e da distribuição das precipitações ao longo do ano, em função, também, da ocorrência de eventos climáticos de grande escala, como El Niño e La Niña.

A equação que correlaciona as características fundamentais de uma chuva intensa é aquela proposta por Chen (1983), dada pela Equação 1 seguinte:

$$
I_{m}=\frac{K \cdot T R^{a}}{(t+b)^{c}} \quad \text { Eq. } 1
$$

em que: $\mathrm{I}_{\mathrm{m}}$ é a intensidade máxima média de precipitação em $\mathrm{mm} \mathrm{h}^{-1}$, TR é o tempo de retorno da chuva em anos e K, a, b, c são parâmetros locais obtidos por regressão não-linear, com base nas informações extraídas de pluviogramas.

Diversos estudos já foram feitos no país para a obtenção das equações $i$ - $d$ - $f$, desde o pioneiro Pfafstetter (1957), passando pelos de Fendrich (1998; 2003), para o estado do Paraná, Pinto (1996; 1999) para o Espírito Santo e Rio de Janeiro respectivamente, Freitas et al (2001) para Minas Gerais, Silva et al (2002) para a Bahia, Silva et al (2003) para o Tocantins e Oliveira et al (2005) para Goiás. Contudo, nem sempre as equações $i$ - $d-f$ estão disponíveis em determinada localidade na qual os projetos de engenharia são conduzidos. Neste caso, de acordo com Pruski et al (2006), podese utilizar da equação obtida de um pluviógrafo situado em localidade próxima que possua clima semelhante, ou, então, interpolar resultados obtidos em localidades próximas ao local de interesse. Essa segunda alternativa foi a empregada no presente artigo.

\section{METODOLOGIA}

Neste estudo, foram estimadas as equações de chuvas intensas para 44 localidades do oeste do estado de Minas Gerais e do entorno do lago do reservatório da Usina Hidrelétrica de Furnas (FIG. 1), compreendidas entre $20^{\circ} 00$ a $21^{\circ} 25^{\prime}$ de latitudes Sul e entre $45^{\circ} 00^{\prime}$ e $46^{\circ} 25^{\prime}$ de longitudes 
Oeste (TAB. 1). A metodologia empregada na determinação dos parâmetros da Eq. 1 foi a do inverso das potências da distância entre as localidades, o que permitiu estimar as chuvas intensas máximas médias (de 5 a 60 minutos, espaçadas de 5 em 5 minutos), em função dos períodos de retorno (de 2 até 10.000 anos).

Devido à inexistência de dados pluviográficos na maioria das localidades, utilizou-se a metodologia proposta por Pruski (2006) que empregou um interpolador que permitiu estimar cada um dos parâmetros, a partir de dados observados de algumas das localidades.

Figura 1 - Mapa do Reservatório da UHE de Furnas, região centro-oeste do estado de Minas Gerais

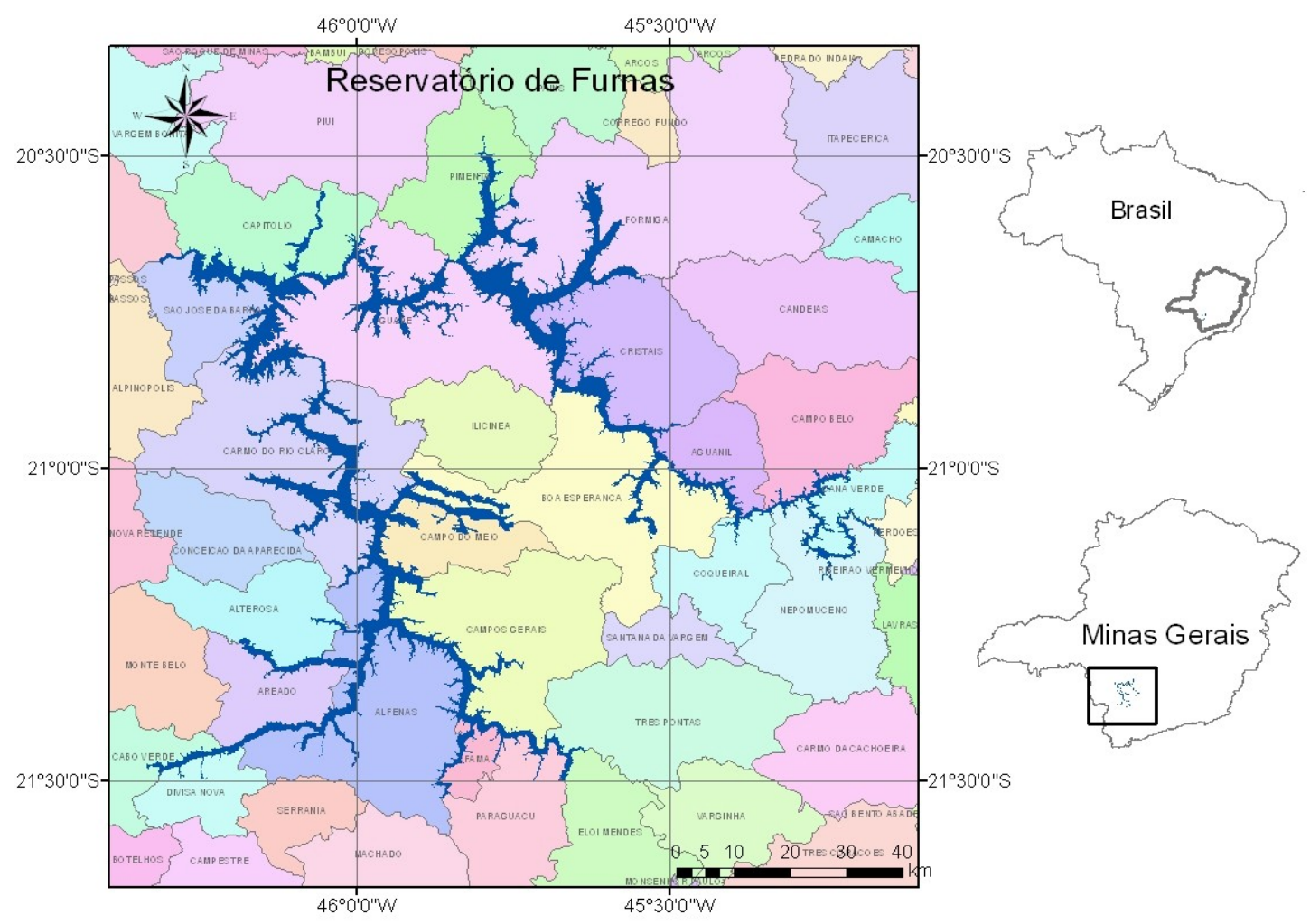

\section{RESULTADOS E DISCUSSÃO}

Na TAB. 1, são apresentadas as coordenadas geográficas para cada uma das 44 localidades em que foram determinadas as equações $i-d-f$ e seus parâmetros. A partir dessas informações, foram estimadas, em cada localidade, as intensidades máximas médias das chuvas $\left(\mathrm{mm} \mathrm{h}^{-1}\right)$, em função do tempo de retorno e de sua duração. Com essas informações, foram elaboradas curvas de distribuição empírica das chuvas intensas com durações de cinco a 60 minutos (em intervalos de cinco em cinco 
minutos) e para tempos de retorno (TR) de 2, 10, 50 e 1000 anos, para 14 localidades da região em estudo (Figuras 2 a 8). Os valores das chuvas intensas apresentados nas curvas podem não representar de maneira fiel as escalas dos gráficos apresentados. Os TR foram definidos em função de sua efetiva aplicabilidade: 1000 anos (para obras de barragens), 50 anos (obras em pequenas bacias urbanas), 10 anos (canais e galerias de águas pluviais) e 2 anos (obras de microdrenagem de caráter residencial). (PRUSKI et al, 2006)

Da mesma forma, foram estimadas as equações de melhor ajuste para cada curva, em cada localidade, juntamente com seus coeficientes de determinação. É importante ressaltar que, buscando uma maior precisão e avaliação do método empregado, tais estimativas precisam ser comparadas com dados pluviográficos observados (onde existirem), com levantamento também da estatística descritiva das séries históricas dessas precipitações.

Pelas figuras apresentadas, como era de se esperar, observa-se que as curvas de distribuição empíricas, em cada localidade, guardam muitas semelhanças. Para todas as curvas, o melhor ajuste se fez por uma função logarítmica, embora os valores pudessem diferir em função da distância do lago do reservatório ou de suas coordenadas geográficas. É bastante provável que boa parte do comportamento das chuvas máximas na região possa sofrer algum grau de influência de efeitos orográficos e de proximidade de corpos d’água. Contudo, tais assunções demandariam outros estudos e pesquisas.

Análises dos eventos por seus tempos de retorno e durações das chuvas permitem observar uma acentuada variabilidade nos valores das precipitações máximas prováveis, em cada localidade. Não se pode precisar, contudo, se a maior ou menor proximidade da massa de água do lago ou fatores como a altitude interferem diretamente na intensidade da precipitação.

Figura 2 - Intensidades máximas das chuvas $\left(\mathrm{mm} \mathrm{h}^{-1}\right)$ em função da duração (minutos) e do tempo de retorno (anos), para Formiga (esquerda) e Pimenta (direita), Minas Gerais. 

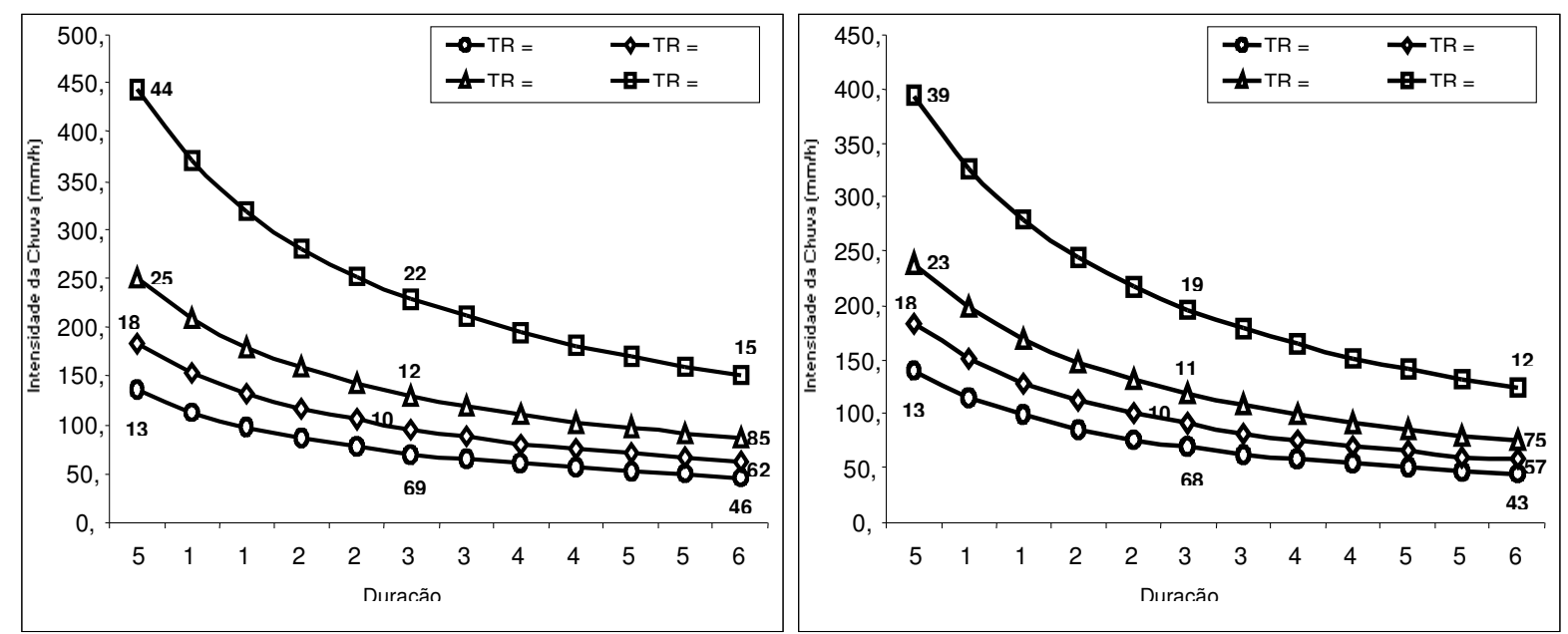

Tabela 1 - Coordenadas geográficas e parâmetros da equação de chuvas intensas para os municípios da Associação dos Municípios do Lago de Furnas (ALAGO) e da região Oeste de Minas Gerais.

\begin{tabular}{|c|c|c|c|c|c|c|c|}
\hline $\begin{array}{c}\text { LOCALIDADES } \\
\text { (ALAGO E OESTE DE MG) }\end{array}$ & $\begin{array}{r}\text { Lat } \\
(\mathbf{S}) \\
\end{array}$ & $\begin{array}{c}\text { Long } \\
(\mathrm{W})\end{array}$ & $\begin{array}{l}\text { Alt } \\
(\mathbf{m})\end{array}$ & $\mathbf{K}$ & $\mathbf{b}$ & $\mathbf{a}$ & c \\
\hline 1. Aguanil & $20^{\circ} 56^{\prime}$ & $45^{\circ} 23^{\prime}$ & 823 & 3643,659 & 29,581 & 0,171 & 1,011 \\
\hline 2. Alfenas & $21^{\circ} 25^{\prime}$ & $45^{\circ} 56^{\prime}$ & 881 & 3810,884 & 20,339 & 0,207 & 1,075 \\
\hline 3. Alpinópolis & $20^{\circ} 51^{\prime}$ & $46^{\circ} 23^{\prime}$ & 876 & 3156,330 & 17,827 & 0,205 & 1,009 \\
\hline 4. Alterosa & $21^{\circ} 14^{\prime}$ & $46^{\circ} 08^{\prime}$ & 843 & 3571,337 & 18,518 & 0,216 & 1,047 \\
\hline 5. Arcos & $20^{\circ} 16^{\prime}$ & $45^{\circ} 32^{\prime}$ & 740 & 826,446 & 10,188 & 0,194 & 0,742 \\
\hline 6. Areado & $21^{\circ} 21^{\prime}$ & $46^{\circ} 08^{\prime}$ & 815 & 3645,200 & 19,111 & 0,213 & 1,056 \\
\hline 7. Bambuí & $20^{\circ} 00^{\prime}$ & $45^{\circ} 58^{\prime}$ & 706 & 978,2050 & 17,83 & 0,187 & 0,722 \\
\hline 8. Boa Esperança & $21^{\circ} 05^{\prime}$ & $45^{\circ} 33^{\prime}$ & 775 & 4291,578 & 31,733 & 0,175 & 1,025 \\
\hline 9. Cabo Verde & $21^{\circ} 28^{\prime}$ & $46^{\circ} 23^{\prime}$ & 927 & 3543,313 & 18,233 & 0,218 & 1,044 \\
\hline 10. Campo Belo & $20^{\circ} 53^{\prime}$ & $45^{\circ} 16^{\prime}$ & 945 & 3628,243 & 29,525 & 0,171 & 1,011 \\
\hline 11. Campo do Meio & $21^{\circ} 06^{\prime}$ & $45^{\circ} 49^{\prime}$ & 785 & 3541,593 & 20,620 & 0,199 & 1,038 \\
\hline 12. Campos Gerais & $21^{\circ} 14^{\prime}$ & $45^{\circ} 45^{\prime}$ & 843 & 3830,386 & 20,705 & 0,206 & 1,071 \\
\hline 13. Cana Verde & $21^{\circ} 01^{\prime}$ & $45^{\circ} 10^{\prime}$ & 867 & 3630,718 & 29,535 & 0,171 & 1,011 \\
\hline 14. Candeias & $20^{\circ} 46^{\prime}$ & $45^{\circ} 16^{\prime}$ & 967 & 3627,415 & 29,519 & 0,171 & 1,011 \\
\hline 15. Capitólio & $20^{\circ} 36^{\prime}$ & $46^{\circ} 03^{\prime}$ & 766 & 2049,092 & 16,674 & 0,168 & 0,913 \\
\hline 16. Carmo do Rio Claro & $20^{\circ} 58^{\prime}$ & $46^{\circ} 07^{\prime}$ & 830 & 2608,310 & 17,324 & 0,186 & 0,961 \\
\hline 17. Cláudio & $20^{\circ} 26^{\prime}$ & $44^{\circ} 45^{\prime}$ & 832 & 692,251 & 9,716 & 0,204 & 0,688 \\
\hline 18. Coqueiral & $21^{\circ} 11^{\prime}$ & $45^{\circ} 26^{\prime}$ & 867 & 5949,010 & 38,665 & 0,179 & 1,059 \\
\hline 19. Córrego Fundo & $20^{\circ} 26^{\prime}$ & $45^{\circ} 33^{\prime}$ & 844 & 940,881 & 10,863 & 0,192 & 0,758 \\
\hline 20. Cristais & $20^{\circ} 52^{\prime}$ & $45^{\circ} 31^{\prime}$ & 873 & 3641,217 & 29,412 & 0,171 & 1,009 \\
\hline 21. Divinópolis & $20^{\circ} 08^{\prime}$ & $44^{\circ} 53^{\prime}$ & 712 & $2.377,021$ & 22,171 & 0,205 & 0,869 \\
\hline 22. Divisa Nova & $21^{\circ} 30^{\prime}$ & $46^{\circ} 11^{\prime}$ & 877 & 3663,250 & 19,489 & 0,210 & 1,059 \\
\hline 23. Elói Mendes & $21^{\circ} 36^{\prime}$ & $45^{\circ} 33^{\prime}$ & 907 & 4262,090 & 23,324 & 0,209 & 1,067 \\
\hline 24. Fama & $21^{\circ} 24^{\prime}$ & $45^{\circ} 49^{\prime}$ & 776 & 3810,506 & 20,341 & 0,207 & 1,075 \\
\hline 25. Formiga & $20^{\circ} 27^{\prime}$ & $45^{\circ} 25^{\prime}$ & 841 & 1320,945 & 14,740 & 0,191 & 0,808 \\
\hline 26. Guapé & $20^{\circ} 45^{\prime}$ & $45^{\circ} 55^{\prime}$ & 760 & 2048,334 & 16,670 & 0,168 & 0,913 \\
\hline 27. Iguatama & $20^{\circ} 10^{\prime}$ & $45^{\circ} 42^{\prime}$ & 664 & 819,687 & 10,121 & 0,194 & 0,741 \\
\hline
\end{tabular}




\begin{tabular}{|l|c|c|c|c|c|c|c|}
\hline 28. Ilicínea & $20^{\circ} 56^{\prime}$ & $45^{\circ} 49^{\prime}$ & 857 & 2126,349 & 17,075 & 0,169 & 0,917 \\
\hline 29. Itapecerica & $20^{\circ} 28^{\prime}$ & $45^{\circ} 07^{\prime}$ & 835 & 734,727 & 10,032 & 0,203 & 0,694 \\
\hline 30. Itaúna & $20^{\circ} 04^{\prime}$ & $44^{\circ} 34^{\prime}$ & 880 & 3481,557 & 31,697 & 0,240 & 0,964 \\
\hline 31. Lavras & $21^{\circ} 14^{\prime}$ & $44^{\circ} 59^{\prime}$ & 919 & 10224,810 & 56,281 & 0,187 & 1,149 \\
\hline 32. Machado & $21^{\circ} 40^{\prime}$ & $45^{\circ} 55^{\prime}$ & 820 & 3811,290 & 20,340 & 0,207 & 1,075 \\
\hline 33. Nepomuceno & $21^{\circ} 14^{\prime}$ & $45^{\circ} 14^{\prime}$ & 840 & 9821,279 & 54,553 & 0,187 & 1,141 \\
\hline 34. Oliveira & $20^{\circ} 41^{\prime}$ & $44^{\circ} 49^{\prime}$ & 982 & 692,260 & 9,716 & 0,204 & 0,688 \\
\hline 35. Pains & $20^{\circ} 22^{\prime}$ & $45^{\circ} 39^{\prime}$ & 693 & 837,842 & 10,219 & 0,194 & 0,744 \\
\hline 36. Paraguaçu & $21^{\circ} 32^{\prime}$ & $45^{\circ} 44^{\prime}$ & 826 & 3810,660 & 20,341 & 0,207 & 1,075 \\
\hline 37. Perdões & $21^{\circ} 05^{\prime}$ & $45^{\circ} 05^{\prime}$ & 842 & 7201,555 & 43,123 & 0,188 & 1,087 \\
\hline 38. Pimenta & $20^{\circ} 29^{\prime}$ & $45^{\circ} 47^{\prime}$ & 776 & 2048,582 & 16,671 & 0,168 & 0,913 \\
\hline 39. Piumhi & $20^{\circ} 27^{\prime}$ & $45^{\circ} 57^{\prime}$ & 793 & 2049,118 & 16,674 & 0,168 & 0,913 \\
\hline 40. Ribeirão Vermelho & $21^{\circ} 11^{\prime}$ & $45^{\circ} 03^{\prime}$ & 808 & 10219,194 & 56,255 & 0,187 & 1,149 \\
\hline 41. Santo Antônio do Monte & $20^{\circ} 05^{\prime}$ & $45^{\circ} 17^{\prime}$ & 919 & 1727,084 & 19,210 & 0,189 & 0,870 \\
\hline 42. São João Batista do Glória & $20^{\circ} 38^{\prime}$ & $46^{\circ} 30^{\prime}$ & 695 & 2122,868 & 16,736 & 0,173 & 0,915 \\
\hline 43. Três Pontas & $21^{\circ} 22^{\prime}$ & $45^{\circ} 30^{\prime}$ & 885 & 5690,461 & 32,626 & 0,200 & 1,080 \\
\hline 44. Varginha & $21^{\circ} 33^{\prime}$ & $45^{\circ} 25^{\prime}$ & 916 & 5987,104 & 32,694 & 0,218 & 1,087 \\
\hline
\end{tabular}

Figura 3 - Intensidades máximas das chuvas $\left(\mathrm{mm} \mathrm{h}^{-1}\right)$ em função da duração (minutos) e do tempo de retorno (anos), para Cristais (esquerda) e Guapé (direita), Minas Gerais.
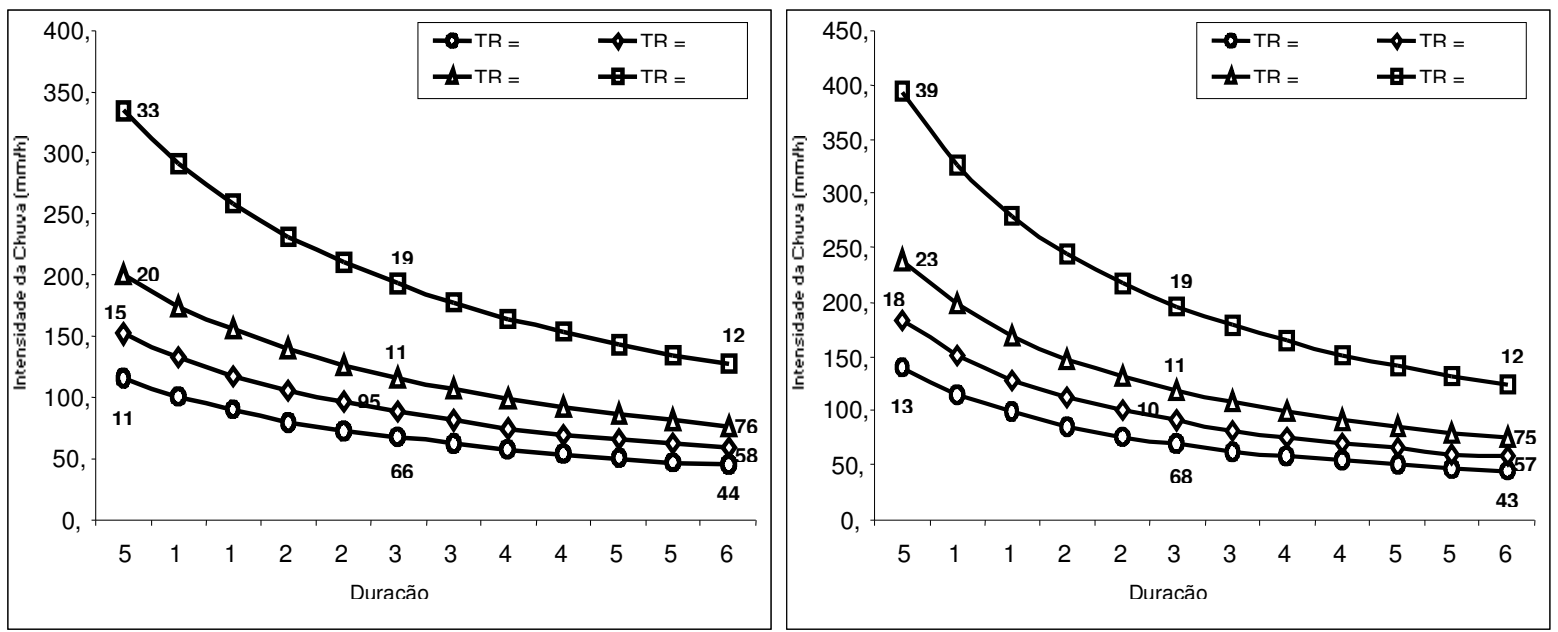

Figura 4 - Intensidades máximas das chuvas $\left(\mathrm{mm} \mathrm{h}^{-1}\right)$ em função da duração (minutos) e do tempo de retorno (anos), para Ribeirão Vermelho (esquerda) e Boa Esperança (direita), Minas Gerais. 

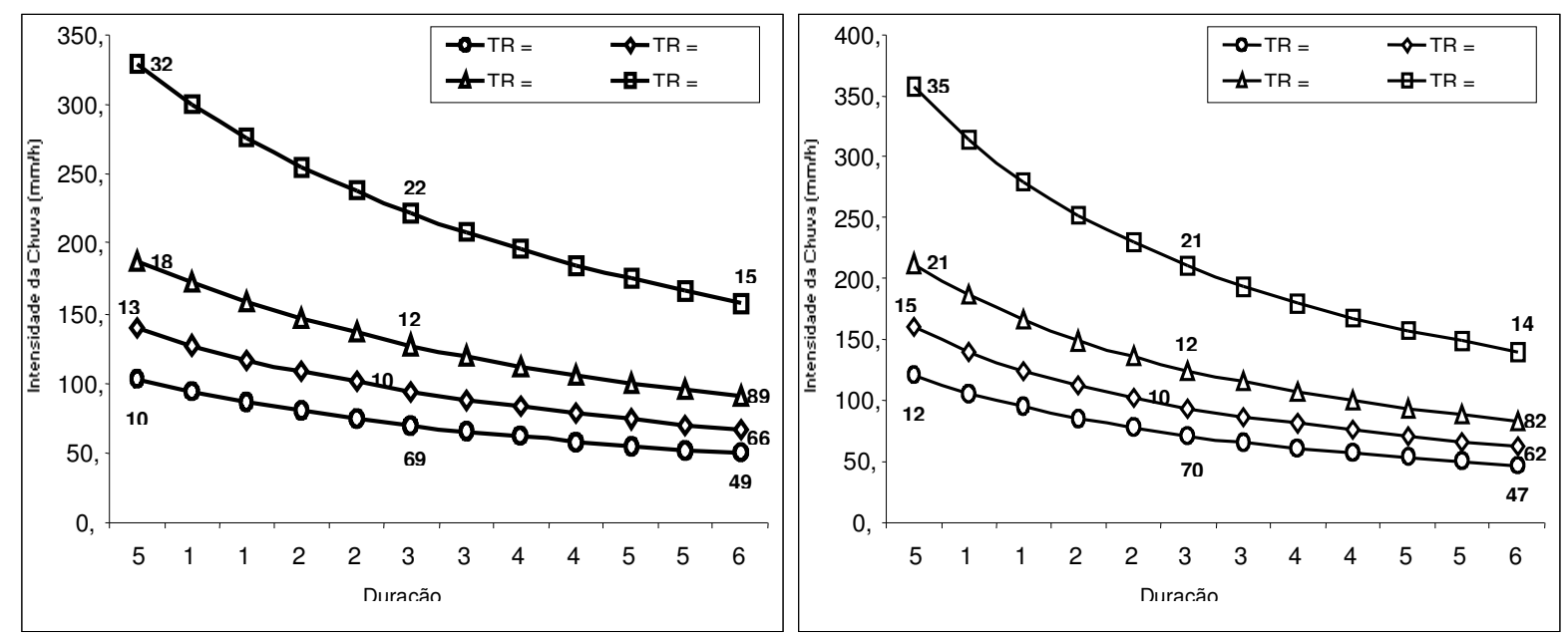

Figura 5 - Intensidades máximas das chuvas $\left(\mathrm{mm} \mathrm{h}^{-1}\right)$ em função da duração (minutos) e do tempo de retorno (anos), para Varginha (esquerda) e São João Batista do Glória (direita), Minas Gerais.
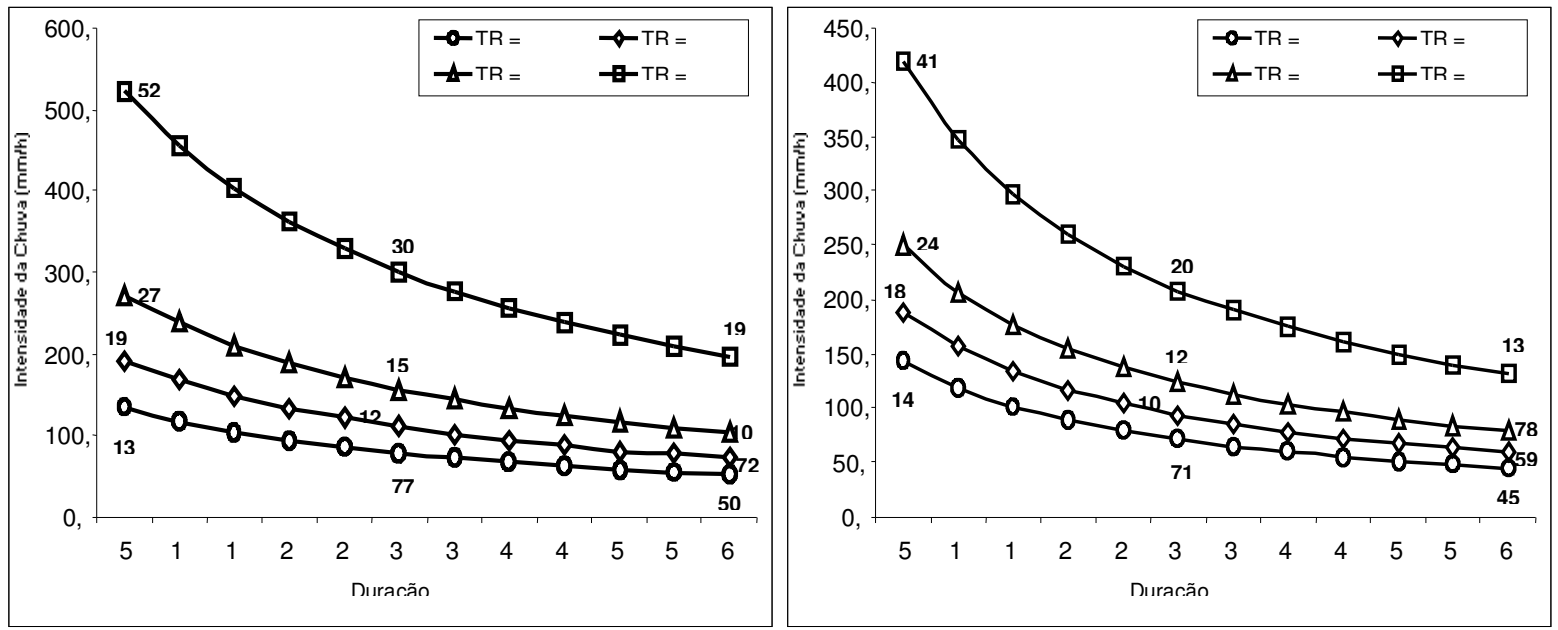

Figura 6 - Intensidades máximas das chuvas $\left(\mathrm{mm} \mathrm{h}^{-1}\right)$ em função da duração (minutos) e do tempo de retorno (anos), para Campo do Meio (esquerda) e Areado (direita), Minas Gerais.
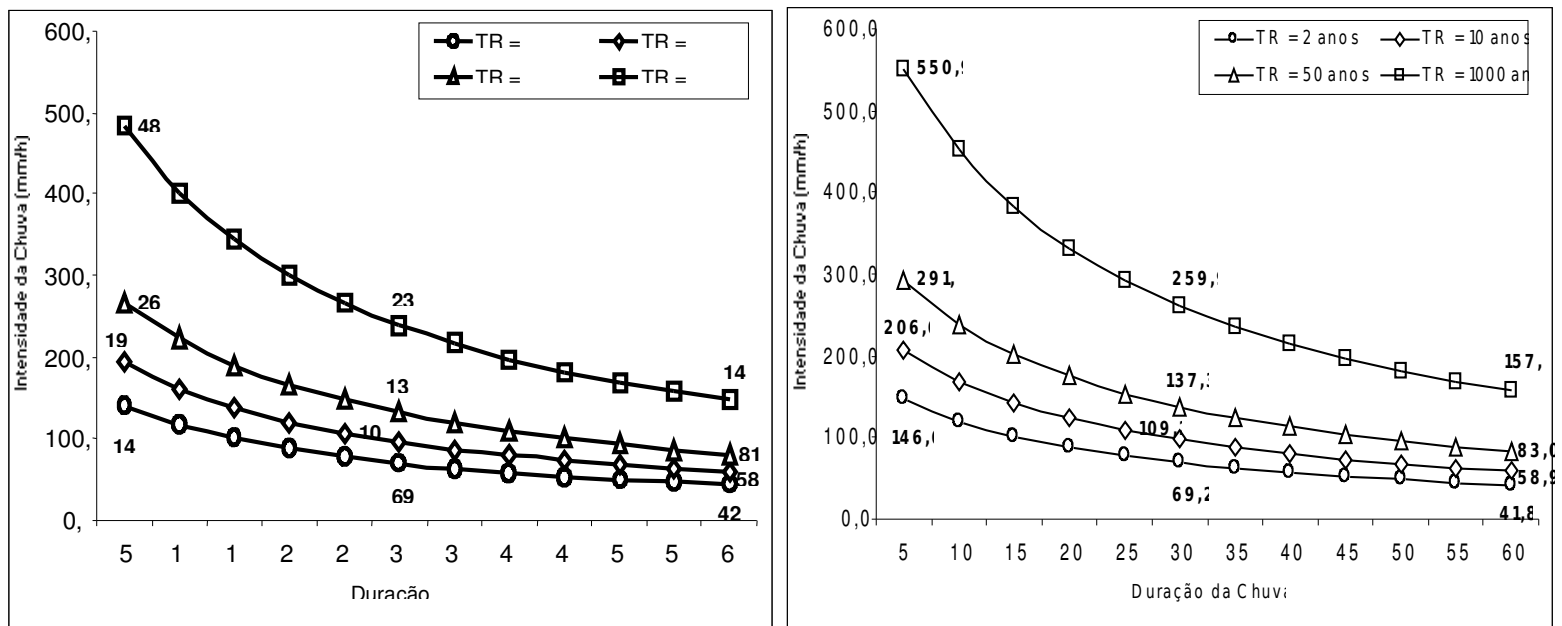
Figura 7 - Intensidades máximas das chuvas $\left(\mathrm{mm} \mathrm{h}^{-1}\right)$ em função da duração (minutos) e do tempo de retorno (anos), para Fama (esquerda) e Carmo do Rio Claro (direita), Minas Gerais.
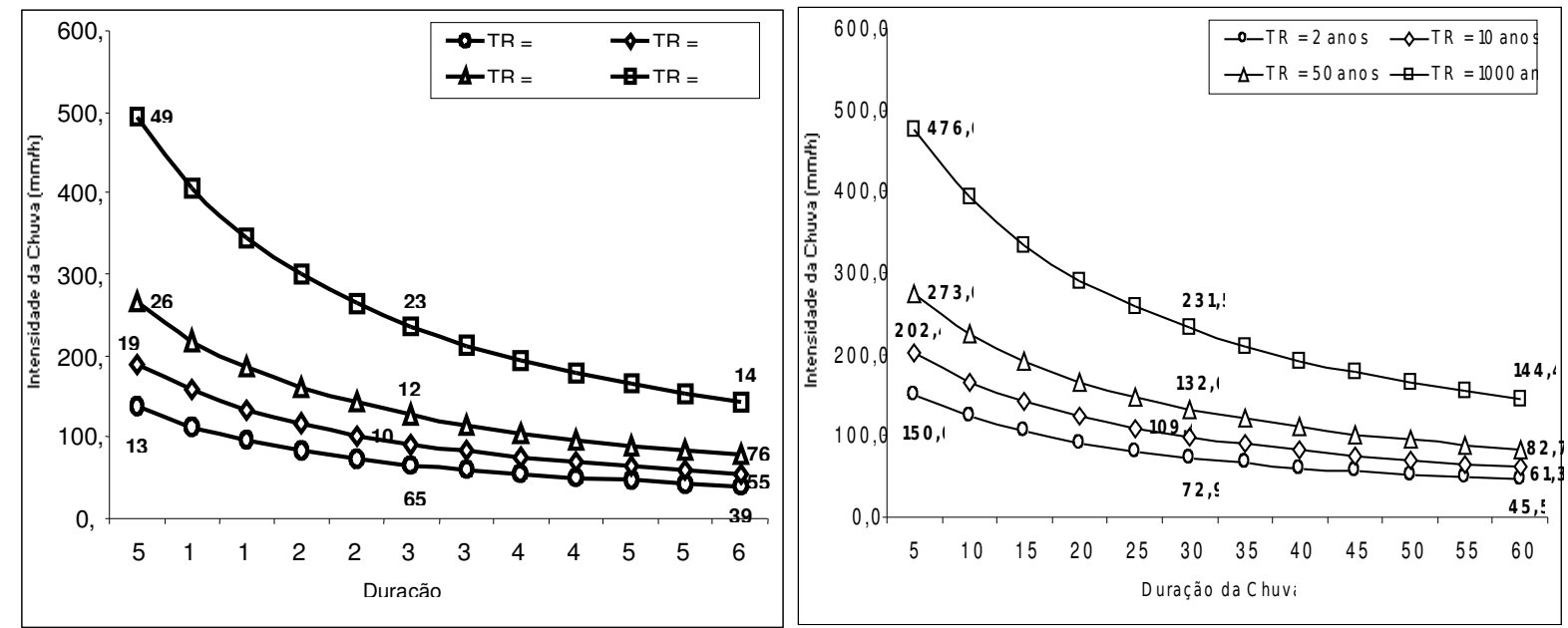
Tabela 2 - Equações logarítmicas de melhor ajuste na determinação das intensidades máximas de chuvas ( $\mathrm{I}_{\mathrm{C}}$ ), mm.h ${ }^{-1}$, em função do tempo de retorno (anos) e da duração das chuvas $\left(\mathrm{D}_{\mathrm{C}}\right)$, em minutos.

\begin{tabular}{|c|c|c|c|c|}
\hline & \multicolumn{4}{|c|}{ TEMPO DE RETORNO (anos) } \\
\hline LOCALIDADE & 2 & 10 & $\mathbf{5 0}$ & 1000 \\
\hline Formiga & $\begin{array}{c}\mathrm{I}_{\mathrm{C}}=- \\
-36,802 \cdot \ln \left(\mathrm{D}_{\mathrm{C}}\right) \\
+136,64 \\
\left(\mathrm{R}^{2}=0,999\right)\end{array}$ & $\begin{array}{c}\mathrm{I}_{\mathrm{C}}=-50,047 \cdot \ln \left(\mathrm{D}_{\mathrm{C}}\right)+185,81 \\
\left(\mathrm{R}^{2}=0,999\right)\end{array}$ & $\begin{array}{c}\mathrm{I}_{\mathrm{C}}=-68,058 \cdot \ln \left(\mathrm{D}_{\mathrm{C}}\right)+252,68 \\
\left(\mathrm{R}^{2}=0,999\right)\end{array}$ & $\begin{array}{c}\mathrm{I}_{\mathrm{C}}=-120,61 \cdot \ln \left(\mathrm{D}_{\mathrm{C}}\right)+447,78 \\
\left(\mathrm{R}^{2}=0,999\right)\end{array}$ \\
\hline Pimenta & $\begin{array}{c}\mathrm{I}_{\mathrm{C}}=-39,231 . \ln \left(\mathrm{D}_{\mathrm{C}}\right)+140,09 \\
\left(\mathrm{R}^{2}=0,998\right)\end{array}$ & $\begin{array}{c}\mathrm{I}_{\mathrm{C}}=-51,411 . \ln \left(\mathrm{D}_{\mathrm{C}}\right)+183,58 \\
\left(\mathrm{R}^{2}=0,998\right)\end{array}$ & $\begin{array}{c}\mathrm{I}_{\mathrm{C}}=-67,372 \cdot \ln \left(\mathrm{D}_{\mathrm{C}}\right)+240,58 \\
\left(\mathrm{R}^{2}=0,998\right)\end{array}$ & $\begin{array}{c}\mathrm{I}_{\mathrm{C}}=-111,44 \cdot \ln \left(\mathrm{D}_{\mathrm{C}}\right)+397,95 \\
\left(\mathrm{R}^{2}=0,998\right)\end{array}$ \\
\hline Cristais & $\begin{array}{c}\mathrm{I}_{\mathrm{C}}=-29,91 \cdot \ell \mathrm{n}\left(\mathrm{D}_{\mathrm{C}}\right)+119,48 \\
\left(\mathrm{R}^{2}=0,993\right)\end{array}$ & $\begin{array}{c}\mathrm{I}_{\mathrm{C}}=-39,386 \cdot \ln \left(\mathrm{D}_{\mathrm{C}}\right)+157,34 \\
\left(\mathrm{R}^{2}=0,993\right)\end{array}$ & $\begin{array}{c}\mathrm{I}_{\mathrm{C}}=-51,864 \cdot \ln \left(\mathrm{D}_{\mathrm{C}}\right)+207,18 \\
\left(\mathrm{R}^{2}=0,993\right)\end{array}$ & $\begin{array}{c}\mathrm{I}_{\mathrm{C}}=-86,565 \cdot \ln \left(\mathrm{D}_{\mathrm{C}}\right)+345,81 \\
\left(\mathrm{R}^{2}=0,993\right)\end{array}$ \\
\hline Guapé & $\begin{array}{c}\mathrm{I}_{\mathrm{C}}=-39,228 . \ell \mathrm{n}\left(\mathrm{D}_{\mathrm{C}}\right)+140,08 \\
\left(\mathrm{R}^{2}=0,998\right)\end{array}$ & $\begin{array}{c}\mathrm{I}_{\mathrm{C}}=-51,407 . \ell \mathrm{n}\left(\mathrm{D}_{\mathrm{C}}\right)+183,57 \\
\left(\mathrm{R}^{2}=0,998\right)\end{array}$ & $\begin{array}{c}\mathrm{I}_{\mathrm{C}}=-67,368 \cdot \ln \left(\mathrm{D}_{\mathrm{C}}\right)+240,56 \\
\left(\mathrm{R}^{2}=0,998\right)\end{array}$ & $\begin{array}{c}\mathrm{I}_{\mathrm{C}}=-111,44 . \ell \mathrm{n}\left(\mathrm{D}_{\mathrm{C}}\right)+397,92 \\
\left(\mathrm{R}^{2}=0,998\right)\end{array}$ \\
\hline $\begin{array}{l}\text { Ribeirão } \\
\text { Vermelho }\end{array}$ & $\begin{array}{c}\mathrm{I}_{\mathrm{C}}=-22,621 \cdot \ln \left(\mathrm{D}_{\mathrm{C}}\right)+108,25 \\
\left(\mathrm{R}^{2}=0,997\right)\end{array}$ & $\begin{array}{c}\mathrm{I}_{\mathrm{C}}=-30,565 \cdot \ln \left(\mathrm{D}_{\mathrm{C}}\right)+146,26 \\
\left(\mathrm{R}^{2}=0,997\right)\end{array}$ & $\begin{array}{c}\mathrm{I}_{\mathrm{C}}=-41,298 . \ell \mathrm{n}\left(\mathrm{D}_{\mathrm{C}}\right)+197,62 \\
\left(\mathrm{R}^{2}=0,997\right)\end{array}$ & $\begin{array}{c}\mathrm{I}_{\mathrm{C}}=-72,314 \cdot \ln \left(\mathrm{D}_{\mathrm{C}}\right)+346,03 \\
\left(\mathrm{R}^{2}=0,977\right)\end{array}$ \\
\hline Boa Esperança & $\begin{array}{c}\mathrm{I}_{\mathrm{C}}=-30,790 \cdot \ell \mathrm{n}\left(\mathrm{D}_{\mathrm{C}}\right)+125,14 \\
\left(\mathrm{R}^{2}=0,991\right)\end{array}$ & $\begin{array}{c}\mathrm{I}_{\mathrm{C}}=-40,806 \cdot \ln \left(\mathrm{D}_{\mathrm{C}}\right)+165,85 \\
\left(\mathrm{R}^{2}=0,991\right)\end{array}$ & $\begin{array}{c}\mathrm{I}_{\mathrm{C}}=-54,081 \cdot \ln \left(\mathrm{D}_{\mathrm{C}}\right)+219,8 \\
\left(\mathrm{R}^{2}=0,991\right)\end{array}$ & $\begin{array}{c}\mathrm{I}_{\mathrm{C}}=-91,353 \cdot \ln \left(\mathrm{D}_{\mathrm{C}}\right)+371,29 \\
\left(\mathrm{R}^{2}=0,991\right)\end{array}$ \\
\hline Varginha & $\begin{array}{c}\mathrm{I}_{\mathrm{C}}=-35,284 \cdot \ln \left(\mathrm{D}_{\mathrm{C}}\right)+139,86 \\
\left(\mathrm{R}^{2}=0,992\right)\end{array}$ & $\begin{array}{c}\mathrm{I}_{\mathrm{C}}=-50,114 \cdot \ell \mathrm{n}\left(\mathrm{D}_{\mathrm{C}}\right)+198,64 \\
\left(\mathrm{R}^{2}=0,992\right)\end{array}$ & $\begin{array}{c}\mathrm{I}_{\mathrm{C}}=-71,176 \cdot \ln \left(\mathrm{D}_{\mathrm{C}}\right)+282,12 \\
\left(\mathrm{R}^{2}=0,992\right)\end{array}$ & $\begin{array}{c}\mathrm{I}_{\mathrm{C}}=-91,353 \cdot \ln \left(\mathrm{D}_{\mathrm{C}}\right)+371,29 \\
\left(\mathrm{R}^{2}=0,992\right)\end{array}$ \\
\hline $\begin{array}{l}\text { S. J. Batista do } \\
\text { Glória }\end{array}$ & $\begin{array}{c}\mathrm{I}_{\mathrm{C}}=-40,447 \cdot \ln \left(\mathrm{D}_{\mathrm{C}}\right)+144,40 \\
\left(\mathrm{R}^{2}=0,998\right)\end{array}$ & $\begin{array}{c}\mathrm{I}_{\mathrm{C}}=-53,433 \cdot \ln \left(\mathrm{D}_{\mathrm{C}}\right)+190,76 \\
\left(\mathrm{R}^{2}=0,998\right)\end{array}$ & $\begin{array}{c}\mathrm{I}_{\mathrm{C}}=-70,588 \cdot \ln \left(\mathrm{D}_{\mathrm{C}}\right)+252,00 \\
\left(\mathrm{R}^{2}=0,998\right)\end{array}$ & $\begin{array}{c}\mathrm{I}_{\mathrm{C}}=-118,53 \cdot \ln \left(\mathrm{D}_{\mathrm{C}}\right)+423,14 \\
\left(\mathrm{R}^{2}=0,998\right)\end{array}$ \\
\hline Campo do Meio & $\begin{array}{c}\mathrm{I}_{\mathrm{C}}=-40,485 \cdot \ln \left(\mathrm{D}_{\mathrm{C}}\right)+142,43 \\
\left(\mathrm{R}^{2}=0,998\right)\end{array}$ & $\begin{array}{c}\mathrm{I}_{\mathrm{C}}=-55,768 \cdot \ln \left(\mathrm{D}_{\mathrm{C}}\right)+196,19 \\
\left(\mathrm{R}^{2}=0,998\right)\end{array}$ & $\begin{array}{c}\mathrm{I}_{\mathrm{C}}=-76,822 \cdot \ln \left(\mathrm{D}_{\mathrm{C}}\right)+270,26 \\
\left(\mathrm{R}^{2}=0,998\right)\end{array}$ & $\begin{array}{c}\mathrm{I}_{\mathrm{C}}=-139,44 \cdot \ln \left(\mathrm{D}_{\mathrm{C}}\right)+490,55 \\
\left(\mathrm{R}^{2}=0,998\right)\end{array}$ \\
\hline Areado & $\begin{array}{c}\mathrm{I}_{\mathrm{C}}=-43,338 \cdot \ln \left(\mathrm{D}_{\mathrm{C}}\right)+141,97 \\
\left(\mathrm{R}^{2}=0,998\right)\end{array}$ & $\begin{array}{c}\mathrm{I}_{\mathrm{C}}=-61,058 . \ln \left(\mathrm{D}_{\mathrm{C}}\right)+298,48 \\
\left(\mathrm{R}^{2}=0,998\right)\end{array}$ & $\begin{array}{c}\mathrm{I}_{\mathrm{C}}=-86,025 \cdot \ln \left(\mathrm{D}_{\mathrm{C}}\right)+293,72 \\
\left(\mathrm{R}^{2}=0,998\right)\end{array}$ & $\begin{array}{c}\mathrm{I}_{\mathrm{C}}=-162,83 \cdot \ell \mathrm{n}\left(\mathrm{D}_{\mathrm{C}}\right)+555,98 \\
\left(\mathrm{R}^{2}=0,998\right)\end{array}$ \\
\hline Fama & $\begin{array}{c}\mathrm{I}_{\mathrm{C}}=-40,099 \cdot \ell \mathrm{n}\left(\mathrm{D}_{\mathrm{C}}\right)+137,87 \\
\left(\mathrm{R}^{2}=0,998\right)\end{array}$ & $\begin{array}{c}\mathrm{I}_{\mathrm{C}}=-55,953 \cdot \ln \left(\mathrm{D}_{\mathrm{C}}\right)+192,38 \\
\left(\mathrm{R}^{2}=0,998\right)\end{array}$ & $\begin{array}{c}\mathrm{I}_{\mathrm{C}}=-78,075 \cdot \ln \left(\mathrm{D}_{\mathrm{C}}\right)+268,44 \\
\left(\mathrm{R}^{2}=0,998\right)\end{array}$ & $\begin{array}{c}\mathrm{I}_{\mathrm{C}}=-145,15 \cdot \ell \mathrm{n}\left(\mathrm{D}_{\mathrm{C}}\right)+499,07 \\
\left(\mathrm{R}^{2}=0,998\right)\end{array}$ \\
\hline
\end{tabular}


Figura 8 - Intensidades máximas das chuvas $\left(\mathrm{mm} \mathrm{h}^{-1}\right)$ em função da duração (minutos) e do tempo de retorno (anos), para Ilicínea (esquerda) e Capitólio (direita), Minas Gerais.
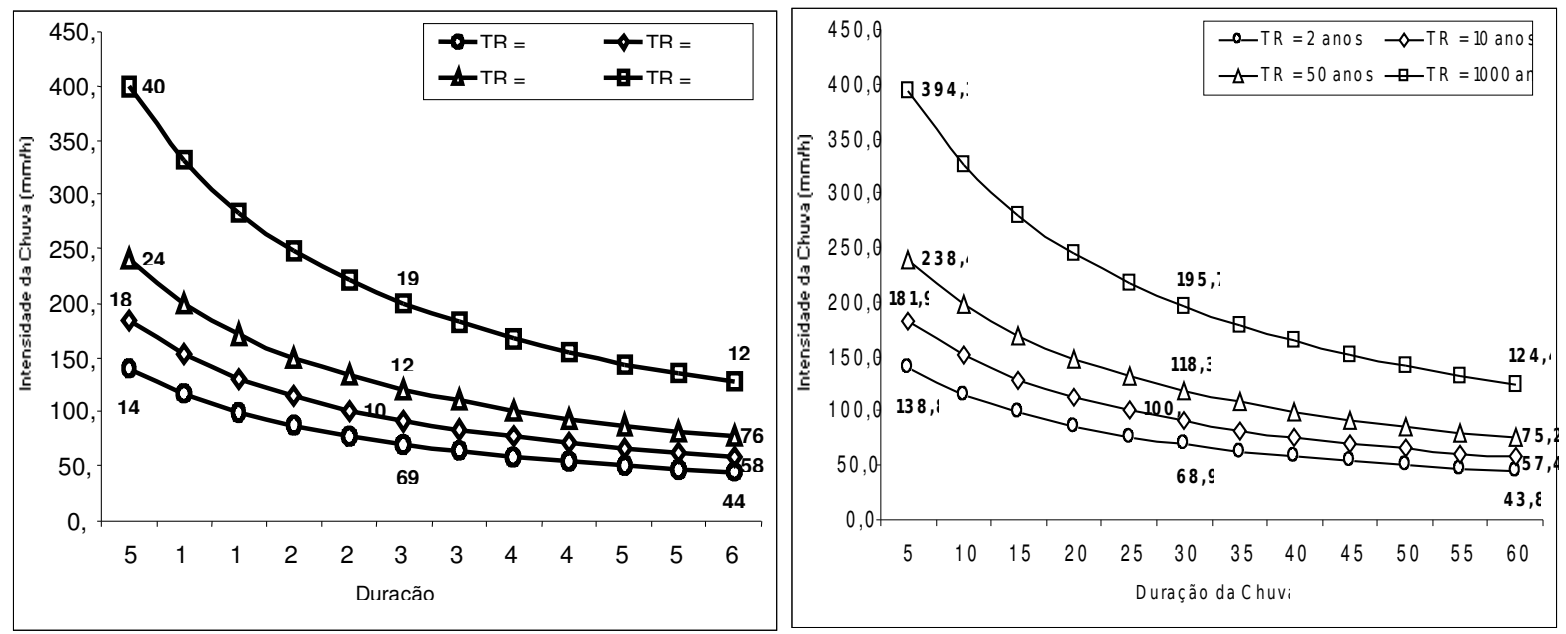

\section{CONCLUSÕES}

A metodologia empregada no presente estudo permitiu gerar equações $i$-d $-f$ (intensidade-duração-frequência) de chuvas, em localidades do oeste do estado de Minas Gerais e do entorno do reservatório da Usina Hidroelétrica de Furnas. Tais equações podem ser valiosas como elementos de projetos de engenharia que levem em conta as relações entre chuvas, vazão de cursos d'água e escoamentos superficiais, com emprego também em dimensionamentos de estruturas hidráulicas. Podem ser úteis também como elemento de apoio aos tomadores de decisão encarregados de elaborar políticas públicas nas áreas de agricultura, saneamento e meio ambiente. Tais estimativas contêm imprecisões próprias do método e devem ser checadas a partir da comparação com séries históricas observadas, pelo emprego de ferramentas estatístico-matemáticas. Tais comparações permitem inferir a compatibilidade entre estimativas empíricas e medidas observadas, buscando obter a correlação entre ambas as variáveis, fornecendo o grau de confiabilidade no método empregado o qual poderá ser utilizado na definição de políticas públicas.

\section{REFERÊNCIAS}

CHEN, C. L. Rainfall intensity-duration-frequency. Journal of hydraulic Engineering, New York, v. 109, n. 12, p. 1603-1621, 1983. 
FENDRICH, R. Chuvas intensas para obras de drenagem no estado do Paraná. Curitiba: Champagnat, 1998.

FENDRICH, R. Chuvas intensas para obras de drenagem no estado do Paraná. 2. ed. Curitiba: Champagnat, 2003.

FREITAS, A. J. D.; SILVA, D. D. D.; PRUSKI, F. F.; PINTO, F. A.; PEREIRA, S. B.; GOMES FILHO, R. R.; TEIXEIRA, A. D. F.; BAENA, L. G. N.; MELLO, L. T. A. D.; NOVAES, L. F. D. Equações de chuvas intensas no estado de Minas Gerais. Belo Horizonte: Copasa/UFV, 2001.

OLIVEIRA, L. F. C. et al. Intensidade - duração - freqüência de chuvas para localidades no Estado de Goiás e Distrito Federal. Pesquisa agropecuária tropical, Goiânia, n. 35, p. 13-18, 2005.

PFAFSTETTER, O. Chuvas intensas no Brasil. Rio de Janeiro: Departamento Nacional de Obras de Saneamento, 1957.

PINTO, F. A. et al. Equações de chuvas intensas para algumas localidades do Estado de Minas Gerais. Engenharia agrícola, Sorocaba, v. 16, n. 1, p. 91-104, 1996.

PINTO, F. A. et al. Equações de intensidade-duração-frequência da precipitação para os estados do Rio de Janeiro e Espírito Santo: estimativa e espacialização. 1999. 70 f. Dissertação (Mestrado em Engenharia Agrícola)-Universidade Federal de Viçosa, Viçosa, MG, 1999.

PRUSKI, F. F.; TEIXEIRA, A. F.; SILVA, D. D.; CECÍLIO, R. A.; SILVA, M. L. N. Dimensionamento de sistemas hidroagrícolas. Viçosa, MG: Ed. UFV, 2006.

SILVA, D. D. et al. Chuvas intensas no Estado da Bahia. Revista brasileira de engenharia agrícola e ambiental, Campina Grande, PB, v. 6, n. 2, p. 362-367, 2002.

SILVA, D. D. et al. Equações de intensidade-duração-frequência da precipitação pluvial para o estado de Tocantins. Engenharia na agricultura, Viçosa, MG, v. 11, n. 1-4, p. 7-14, 2003.

TUCCI, C. E. M. Hidrologia: ciência e aplicação. 3. ed. Porto Alegre: UFRGS/ABRH, 2004. 